\title{
Variabilidade espaço-temporal das cargas dissolvidas de rios em área de proteção ambiental no nordeste brasileiro
}

\section{Spatio-temporal variability of dissolved loads in rivers in environmental protected areas in northeast Brazil}

\author{
Camila dos Santos Brandãoํㅜㄴ Lenilda Pita da Silva ${ }^{2}$; Thais Carvalho Cerqueira Chaussê ${ }^{3}$; Mauricio S. Moreau ${ }^{4}$ \\ e Daniela Mariano Lopes da Silva ${ }^{5}$
}

\author{
1,2,3,5 Universidade Estadual de Santa Cruz, Departamento de Ciências Biológicas, Ilhéus/BA, Brasil \\ milab_biologia@yahoo.com.br; lenildaps@gmail.com; thaiscerqueira2010@yahoo.com.br; dmlsilva@gmail.com \\ ${ }^{4}$ Universidade Estadual de Santa Cruz, Departamento de Ciências Agrárias e Ambientais, Ilhéus/BA, Brasil \\ mmoreau@uesc.br
}

Recebido: 14/07/14 - Revisado: 21/02/15 - Aceito:26/05/15

\section{RESUMO}

O presente trabalho teve por objetivo determinar a variação espaço-temporal e o aporte fluvial dos nutrientes inorgânicos e ions maiores em rios e riachos localizados na bacia bidrográfica do Rio Tijuipe, em uma área de preservação ambiental no Nordeste brasileiro. As amostras de água foram coletadas mensalmente de agosto de 2010 a julbo de 2011, em 11 pontos ao longo da bacia bidrográfica, sendo analisados os ions $\mathrm{NO}_{3}, \mathrm{NO}_{2}, \mathrm{NH}_{4}^{+}, \mathrm{PO}_{4}^{3-}, \mathrm{SiO}_{2}$, $\mathrm{Cl} ; \mathrm{SO}_{4}^{2-}, \mathrm{HCO}_{3}, \mathrm{Na}^{+}, \mathrm{K}^{+}, \mathrm{Mg}^{2+}$ e $\mathrm{Ca}^{2+}$. A presença da mata ciliar no entorno da bacia foi determinante para o comportamento dos nutrientes nos rios sendo que o nitrato foi a forma predominante de nitrogênio inorgânico, o que é esperado em áreas preservadas. Os ions maiores apresentaram a seguinte ordem de grandeza: $\mathrm{K}^{+}<\mathrm{SO}_{4}{ }^{2}<\mathrm{Ca}^{2}+<\mathrm{HCO}_{3}<\mathrm{Mg}^{2}+<\mathrm{Na}^{+}<\mathrm{Cl}$; sendo a maior contribuição relacionada aos ions $\mathrm{Cl}^{-e} \mathrm{Na} \mathrm{N}^{+}$, cuja principal fonte é a deposição atmosférica devido à proximidade com o oceano. Com este trabalho observou-se que a bacia bidrográfica do Rio Tijuipe apresenta características de áreas preservadas, não apresentando variações significativas nas concentrações de ions.

Palavras Chave: Nutrientes. Íons maiores. Mata Atlântica

\begin{abstract}
The aim of this work was to determine the spatio-temporal variation of inorganic nutrients and major ions and their fuxes in Tijuipe River Basinlocated in a conservation area in northeast Brazil. Water samples were collected monthly from August 2010 to July 2011 at 11 sites throughout the watershedand it was analyzed the ions $\mathrm{NO}_{3}, \mathrm{NO}_{2}, \mathrm{NH}_{4}^{+}, \mathrm{PO}_{4}^{3}, \mathrm{SiO}_{2}, \mathrm{Cl} ; \mathrm{SO}_{4}^{2-}, \mathrm{HCO}_{3}, \mathrm{Na}^{+}, \mathrm{K}^{+}, \mathrm{Mg}^{2+}$ and $\mathrm{Ca}^{2+}$. The presence of riparian vegetation was crucial to the behavior of nutrients fluxes and nitrate was the main form in all sites as expected to preserved areas. Major ions were found in the following order of magnitude $\mathrm{K}^{+}<\mathrm{SO}_{4}^{2-}<\mathrm{Ca}^{2}+<\mathrm{HCO}_{3}<\mathrm{Mg}^{2}+<\mathrm{Na}^{+}<\mathrm{Cl}^{-}$, being the largest export related to $\mathrm{Cl}^{-}$and $\mathrm{Na}^{+}$due to atmospheric deposition and the ocean proximity. Based on these results it can be concluded that Tijuipe River Basin could be considered a preserved watershed did not presenting significative differences on ion concentrations between studied rivers.
\end{abstract}

Keywords: Nutrients. Major ions. Atlantic forest 


\section{INTRODUÇÃO}

A Mata Atlântica é a segunda floresta mais ameaçada no mundo, sendo considerada uma das prioridades para a conservação da biodiversidade. É considerada um dos 34 hotspots mundiais devido a seu alto grau de endemismos e ameaças de extinções iminentes (GALINDO-LEAL; CÂMARA, 2005; MYERS et al., 2000). Com a inclusão do Estado do Piauí em 2012 , este bioma passou a apresentar $8,5 \%$ da cobertura original de acordo com o último relatório do SOS Mata Atlântica (2013). No Estado da Bahia, a Mata Atlântica apresenta progressiva devastação, sendo que dos $36 \%$ que cobriam o território baiano originalmente, hoje restam menos de $6 \%$, de forma bastante fragmentada (PNUMA, 2012).

A exploração predatória e o risco de extinção de inúmeras espécies levaram o poder público, a partir de 1950, a adotar medidas legais para proteger o que restava das áreas de floresta nativa. Para tanto, foram criadas as Unidades de Conservação - UCs, para proteger não apenas os recursos bióticos, mas também conservar os recursos físicos e culturais. Ao proteger as florestas, estas áreas de preservação estão protegendo também a manutenção do ciclo hídrico, por agir diretamente no regime de chuvas da região. Diversos estudos mostram que alterações no uso do solo têm influência sobre o ciclo hidrológico e os processos biogeoquímicos que ocorrem na bacia hidrográfica (DEEGAN et al., 2011; DEFRIES; ESHLEMAN, 2004; HOWARTH et al., 2000; PARRON; BUSTAMANTE; MARKEWITZ, 2011; SYVITSKI et al., 2005).

Na Mata Atlântica da Bahia existem aproximadamente 32 UCs, dentre elas, a APA Baía de Camamu, APA de Coroa Vermelha, Jardim Botânico de Salvador, Estação Ecológica Federal Pau Brasil e Parque Estadual da Serra do Conduru. O Parque Estadual da Serra do Conduru (PESC) está localizado no sul do Estado da Bahia e apresenta uma grande relevância para a fauna e a flora desta unidade de conservação com todo o seu entorno englobado por Áreas de Proteção Ambiental (APA). Criado em 1997, o PESC está localizado entre os municípios de Ilhéus, Uruçuca e Itacaré, sendo de grande importância para os dois últimos municípios, por abranger as nascentes dos rios da bacia hidrográfica do Rio Tijuipe, que drenam estes municípios, possuindo uma área de drenagem de aproximadamente 9.275 ha (BRASIL, 1993).

Em vista da importância de estudos sobre as variações que ocorrem na biogeoquímica das bacias com pouca ou nenhuma interferência humana e da possibilidade de estes servirem como referência para estudos posteriores desenvolvidos na região, o presente trabalho teve como objetivo determinar a variação espaço-temporal e o aporte fluvial dos nutrientes inorgânicos e íons maiores em rios pertencentes à Bacia Hidrográfica do Rio Tijuipe (Uruçuca - Itacaré/Bahia).

\section{MATERIAIS E MÉTODOS}

\section{Área de estudo}

A Bacia Hidrográfica do Tijuipe situa-se no sul do
Estado da Bahia, com uma área de 102,34 $\mathrm{Km}^{2}$. Esta bacia está inserida na área da APA Itacaré-Serra Grande e do Parque Estadual Serra do Conduru (IESB, 2005) (Figura 1).

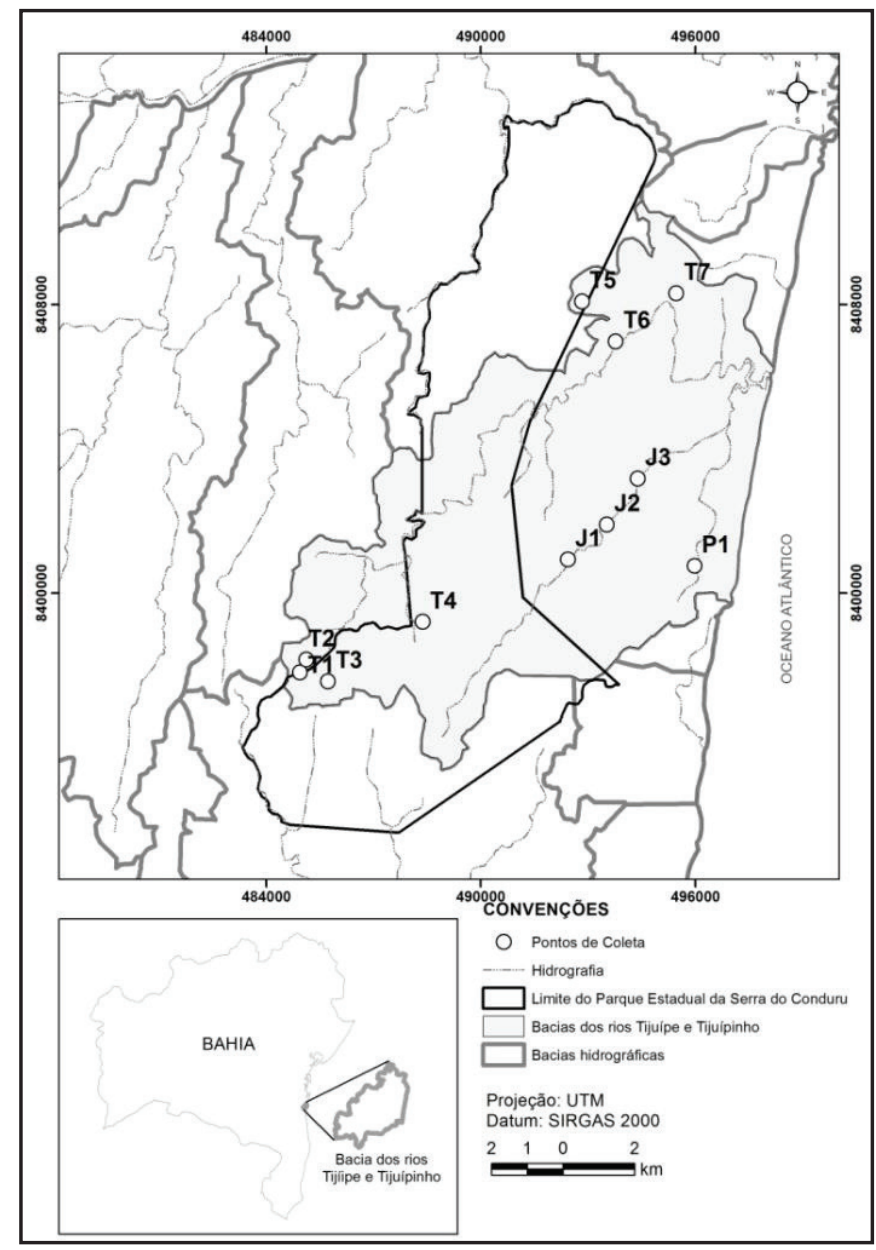

Figura 1 - Pontos de coleta na Bacia hidrográfica do rio Tijuipe em Uruçuca, Bahia (T1 a T7 - Rio Tijuipe; J1 a J3 - Rio Tijuipinho; P1 - Rio Pancadinha)

O clima da região é do tipo Af, conforme o sistema de classificação de Köppen. É um clima quente e úmido, sem estação seca definida, com temperatura média anual em torno de $24^{\circ} \mathrm{C}$. A precipitação pluviométrica é superior a $1.300 \mathrm{~mm}$ anuais bem distribuídos e as máximas pluviométricas ocorrem, de modo geral, no período de fevereiro a julho (ASMAR; ANDRADE, 1977; SÁ; ALMEIDA; LEÃO et al., 1982) (Figura 2). Para determinar os meses secos e chuvosos neste estudo, utilizou-se como parâmetro valores de chuva de $90 \mathrm{~mm}$, com os meses superiores considerados chuvosos (agosto, fevereiro, março, abril, maio e julho) e os inferiores como meses secos (setembro, outubro, novembro, dezembro, janeiro e junho).

Os solos na área da bacia variam da nascente até a foz, do Latossolo vermelho-amarelo Distrófico típico, Latossolo vermelho-amarelo distroférrico típico petroplíntico, o Cambiossolo Háplico distrófico e neossolo (IESB, 2005). Segundo Silva (2012), os solos Latossolos vermelho-amarelo desta região, encontrados na maior parte da bacia, tem o predomínio de areia $(49 \%)$, seguida por silte $(35 \%)$ e argila $(16 \%)$. 
A formação vegetal na área do PESC é a Floresta Ombrófila Densa Submontana, com a maior porcentagem da área $(56,5 \%)$ representada por Vegetação Secundária, em estágio "Avançado" de regeneração. A vegetação presente ao longo dos pontos de coleta do rio Tijuipe é do tipo mata secundária em estágio avançado e médio de regeneração além de áreas sob o sistema agroflorestal cacau-cabruca e áreas de pastagem. Já o rio Tijuipinho apresenta maior parte da sua vegetação como mata secundária em estágio inicial e médio de regeneração e como sistema agroflorestal cacau-cabruca. A área do entorno do ponto de coleta do rio Pancadinha apresenta-se como uma zona urbanizada, com a vegetação existente sendo caracterizada como pasto ou área aberta.

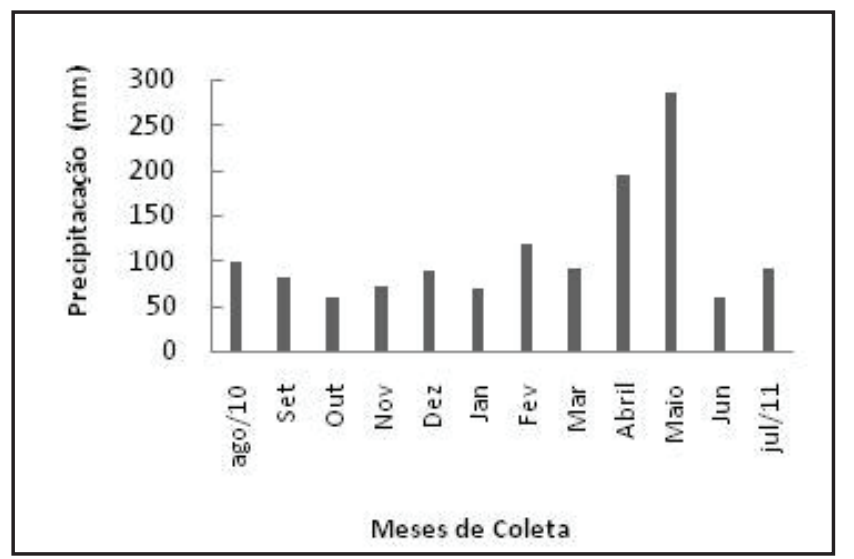

Figura 2 - Precipitação acumulada de 30 dias antes de cada coleta nos meses de agosto/2010 a julho/2011 no município de Itacaré/BA Fonte: PROCLIMA, 2012

\section{PROCEDIMENTO ANALÍTICO}

As coletas das amostras de água foram realizadas mensalmente durante o período de agosto de 2010 a julho de 2011. Foram selecionados 11 pontos ao longo da bacia do Rio Tijuipe sendo os pontos T1-T7 localizados no Rio Tijuipe, o maior rio da bacia, os pontos J1-J3 no Rio Tijuipinho e P1 no Rio Pancadinha. Todos os pontos do rio Tijuipe e do Tijuipinho estão localizados dentro da área da APA Itacaré-Serra Grande, exceto o ponto no rio Pancadinha que é o único que está localizado fora da área da APA apresentando residências em suas margens (Figura 1 e Tabela 1).

Em campo foram medidos temperatura, $\mathrm{pH}$, condutividade elétrica e oxigênio dissolvido utilizando equipamentos portáteis ( $\mathrm{pH} /$ Cond. 340i WTW). As amostras foram filtradas utilizando filtros de fibra de vidro (porosidade $0,7 \mu \mathrm{m}$ ) previamente calcinados a $450{ }^{\circ} \mathrm{C}$ e pesados. O filtrado foi congelado em frascos de polietileno e as análises foram realizadas em um prazo máximo de um mês. O Sólidos Suspensos Totais (SST) foram determinados através da gravimetria após secagem por 24 horas em estufa a $60^{\circ} \mathrm{C}$ e posterior pesagem dos filtros.

A análise das formas inorgânicas dissolvidas $\left(\mathrm{Na}^{+}\right.$,

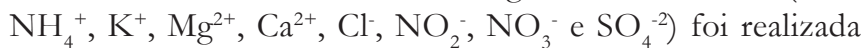
através da cromatografia iônica (DIONEX ICS1000) (PFAFF; HAUTMAN; MUNCH, 1997). O fósforo reativo solúvel $\left(\mathrm{PO}_{4}{ }^{3+}\right)$ e o silicato $\left(\mathrm{SiO}_{2}\right)$ foram determinados por espectrofotometria nos comprimentos de ondas de 880 e $820 \mathrm{~nm}$, respectivamente (CARMOUZE, 1994; GRASSHOFF; EHRARDT; KREMLING, 1983). A concentração do íon bicarbonato $\left(\mathrm{HCO}_{3}^{-}\right)$foi calculada por um modelo de associações iônicas utilizando o fósforo, silicato, temperatura, alcalinidade e $\mathrm{pH}$ com o auxílio do programa CO2SYS.EXE (LEWIS; WALLACE, 1998).

Tabela 1 - Pontos amostrados ao longo da bacia hidrográfica do rio Tijuipe e suas coordenadas geográficas

\begin{tabular}{ccc}
\hline $\begin{array}{c}\text { Pontos de } \\
\text { Coleta }\end{array}$ & \multicolumn{2}{c}{$\begin{array}{c}\text { Coordenadas } \\
\text { Geográfica (UTM) }\end{array}$} \\
\hline T1 & 484948 & 8397781 \\
T2 & 485128 & 8398129 \\
T3 & 485731 & 8397529 \\
T4 & 488371 & 8399179 \\
T5 & 492831 & 8408071 \\
T6 & 493781 & 8406961 \\
T7 & 495469 & 8408301 \\
J1 & 492439 & 8400907 \\
J2 & 493531 & 8401880 \\
J3 & 494387 & 8403156 \\
P1 & 495983 & 8400731 \\
\hline
\end{tabular}

Todas as variáveis analisadas (parâmetros abióticos, nutrientes e íons maiores) foram submetidas ao teste de normalidade Kolmogorov-Smirnov $(\mathrm{p}<0,05)$ e como nenhuma delas apresentou distribuição normal foi então aplicado o teste não paramétrico de Kruskal-Wallis para verificar as diferenças existentes para as variáveis em cada ponto de coleta. Para verificar como os pontos de coleta se distribuem temporal e espacialmente foi realizado a Análise de Componentes Principais (ACP) e foi empregado o teste U de Mann-Whitney para determinar as possíveis diferenças temporais existentes entre os períodos seco e chuvoso para os elementos analisados.

\section{RESULTADOS}

As variáveis abióticas $\mathrm{pH}$ e sólidos suspensos totais (SST) não apresentaram variações significativas entre os rios amostrados, através do teste de Kruskal-Wallis. Os maiores valores de condutividade foram encontrados no Rio Tijuipinho e Pancadinha, não ultrapassando $51 \mu \mathrm{S} \mathrm{cm} \mathrm{cm}^{-1}(\mathrm{p}<0,05)$. O oxigênio dissolvido variou de 80 a $97 \%$ nos pontos T1 e T7, respectivamente $(p<0,05)$, não sendo observadas diferenças entre os rios Tijuipe, Tijuipinho e Pancadinha (Tabela 2).

Em relação ao nitrogênio inorgânico dissolvido (NID), o nitrato foi a forma predominante em relação ao amônio e ao nitrito em toda a bacia. As maiores concentrações de nitrato foram encontradas nos pontos que correspondem as nascentes do Rio Tijuípe que correspondem aos pontos T1 onde as concentrações variaram de 6,6 a 19,4 $\mu \mathrm{M}$ e T2 variando de 7,8 a $28,9 \mu \mathrm{M}$. Nos pontos de J1 a J3 observa-se um aumento nos valores de nitrato no sentido montante-jusante, com valores no ponto J1 que não ultrapassam 1,4 $\mu \mathrm{M}(\mathrm{p}<0,05)$ (Figura 3). 
Tabela 2 - Valores de condutividade (Cond.), pH, saturação de oxigênio (OD \%) e sólidos suspensos totais (SST) nos pontos na bacia hidrográfica do rio Tijuipe (Média e \pm Desvio)

\begin{tabular}{ccccc}
\hline & $\begin{array}{c}\text { Cond. } \\
\left(\boldsymbol{\mu S} / \mathbf{c m}^{-1}\right)\end{array}$ & pH & $\begin{array}{c}\text { OD } \\
(\%)\end{array}$ & $\begin{array}{c}\text { SST } \\
\left(\mathbf{m g ~ L}^{-1}\right)\end{array}$ \\
\hline T1 & $36,7 \pm 3,3^{\mathrm{a}}$ & $5,6 \pm 0,3$ & $80,1 \pm 9,0^{\mathrm{a}}$ & $1,78 \pm 2,3$ \\
T2 & $37,1 \pm 3,0^{\mathrm{a}}$ & $6,3 \pm 0,5$ & $85,3 \pm 9,7$ & $0,99 \pm 0,4$ \\
T3 & $34,7 \pm 3,3^{\mathrm{a}, \mathrm{c}}$ & $5,7 \pm 0,4$ & $78,3 \pm 10,9^{\mathrm{a}, \mathrm{c}}$ & $1,28 \pm 0,7$ \\
T4 & $38,2 \pm 4,2^{\mathrm{a}}$ & $6,1 \pm 0,5$ & $91,4 \pm 10,4$ & $1,83 \pm 1,8$ \\
T5 & $36,3 \pm 3,6^{\mathrm{a}}$ & $6,0 \pm 0,4$ & $96,1 \pm 9,6^{\mathrm{d}}$ & $1,08 \pm 0,5$ \\
T6 & $38,4 \pm 3,9^{\mathrm{a}}$ & $6,0 \pm 0,5$ & $95,4 \pm 9,8$ & $1,80 \pm 0,9$ \\
T7 & $37,5 \pm 3,7^{\mathrm{a}}$ & $6,0 \pm 0,5$ & $97,1 \pm 10,2^{\mathrm{b}}$ & $1,32 \pm 0,7$ \\
J1 & $41,2 \pm 3,7^{\mathrm{d}}$ & $5,9 \pm 0,4$ & $84,5 \pm 14,9$ & $1,31 \pm 2,0$ \\
J2 & $39,9 \pm 4,7^{\mathrm{d}}$ & $6,0 \pm 0,4$ & $91,5 \pm 11,7$ & $1,85 \pm 1,2$ \\
J3 & $40,4 \pm 3,8^{\mathrm{d}}$ & $6,1 \pm 0,4$ & $91,3 \pm 9,3$ & $2,67 \pm 2,0$ \\
P1 & $51,5 \pm 7,2^{\mathrm{b}}$ & $6,0 \pm 0,4$ & $90,7 \pm 12,3$ & $3,92 \pm 1,7$ \\
\hline
\end{tabular}

*Letras diferentes representam diferenças significativas $(\mathrm{p}<0,05)$
O íon amônio apresentou os maiores valores nos pontos T5 e P1, correspondendo aos rios Tijuipe e Pancadinha respectivamente (Figura 3). O nitrito esteve abaixo do limite de detecção do método em todas as campanhas $(1 \mu \mathrm{M})$.

O fosfato apresentou baixa concentração comparado aos demais nutrientes, com valores médios inferiores a $0,3 \mu \mathrm{M}$. Já o silicato apresentou menores concentrações nos pontos a montante de cada rio, sendo mais evidente para o rio Tijuipinho. Além disso, existe uma redução entre os pontos T2 e T4 para o silicato, sendo que estes dois pontos diferiram significativamente entre si $(\mathrm{p}<0,05)$ (Figura 3).

As concentrações dos íons maiores apresentaram a seguinte ordem de grandeza $\mathrm{K}^{+}<\mathrm{SO}_{4}^{2-}<\mathrm{Ca}^{2+}<\mathrm{HCO}_{3}^{-}<$ $\mathrm{Mg}^{2+}<\mathrm{Na}^{+}<\mathrm{Cl}^{-}$sendo os íons cloreto e sódio predominantes nos três rios amostrados, não sendo observado um padrão de aumento ou diminuição no sentido montante-jusante (Figura 4). No geral, não foram encontradas diferenças significativas entre os pontos de coleta para os íons maiores, com exceção do cálcio e do bicarbonato $(\mathrm{p}<0,05)$.

As razões $\mathrm{Cl} / \mathrm{Na}$ variaram de 0,84 a 1,31 para os pontos J2 e T7, respectivamente, com uma média geral de 1,09 \pm 0,12, sendo que os pontos T1, T7 e P1 (1,19, 1,31 e 1,18 respectiva-
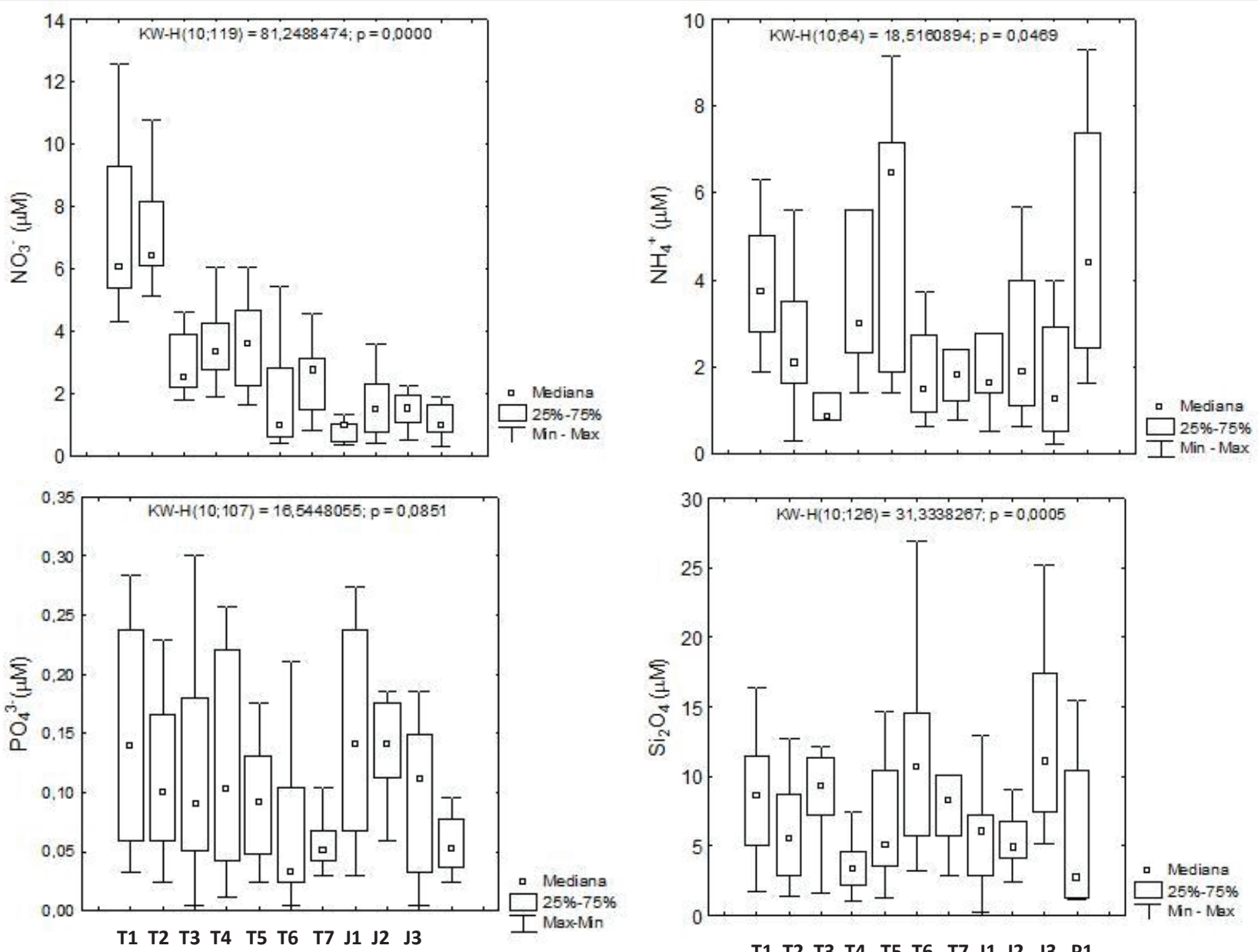

T1 $\begin{array}{llllllllll}\text { T2 } & \text { T3 } & \text { T4 } & \text { T5 } & \text { T6 } & \text { T7 J1 J2 J3 } & \text { P1 }\end{array}$

Figura 3 - Distribuição espacial dos nutrientes $\mathrm{NO}_{3}^{-}, \mathrm{NH}_{4}^{+}, \mathrm{PO}_{4}^{3-} \mathrm{e} \mathrm{Si}_{2} \mathrm{O}_{4}$, ao longo dos pontos de coleta na bacia hidrográfica do rio Tijuipe (Uruçuca, Bahia) 
mente) apresentaram razão média acima da água do mar $(1,17)$. Em relação à Análise do Componente Principal (ACP), os dois primeiros eixos explicam $59,1 \%$ da variância total dos dados, com o eixo 1 apresentando 34,8\% (auto vetor 5,56) e correlação positiva com as variáveis $\mathrm{SO}_{4}^{2-}, \mathrm{Na}^{+}, \mathrm{K}^{+}, \mathrm{Mg}^{2+}$ e Cl, e o eixo 2 apresentando $24,3 \%$ da variância (auto vetor 3,88), com correlação positiva com a condutividade e o Ca2+ (Figura 5).

Os pontos amostrados encontram-se divididos, com a estação seca no lado direito da ACP (quadrantes II e III), sofrendo maior influência dos íons maiores $\mathrm{Na}^{+}, \mathrm{SO}_{4}^{2-}$ e $\mathrm{Cl}^{-}$e a estação chuvosa no lado esquerdo (quadrantes I e IV), com maior influência do OD\% e $\mathrm{HCO}_{3}^{-}$(Figura 5).

Os pontos a montante de cada rio (T1, T2, T3 e J1) para ambas as estações se situaram nos quadrantes inferiores (quadrantes III e IV), com o nitrato tendo maior influência sobre estes pontos no período de seca. Vale ressaltar que o T2 (cheia e seca) é o ponto mais negativos para o eixo 1 , sendo influenciado negativamente pelo $\mathrm{Ca} 2+\mathrm{e}$ a condutividade (Figura 5).

O teste de Mann-Whitney mostrou que entre os nutrientes apenas o nitrato no ponto T4, e o fosfato no ponto T3, apresentaram diferenças significativas entre as estações de seca e de chuva $(p<0,05)$. Para os íons maiores houve diferença significativa entre as estações de seca e de chuva para o $\mathrm{Mg} 2+$ nos pontos T1, T2, T3, T6, J2 e P1; para o SO42- nos pontos T3, T4, T5, T6, J3 e para o Cl- nos pontos T1, T4, T5, T6, J3; e para o $\mathrm{K}+$ apenas no ponto $\mathrm{T} 1(\mathrm{p}<0,05)$.

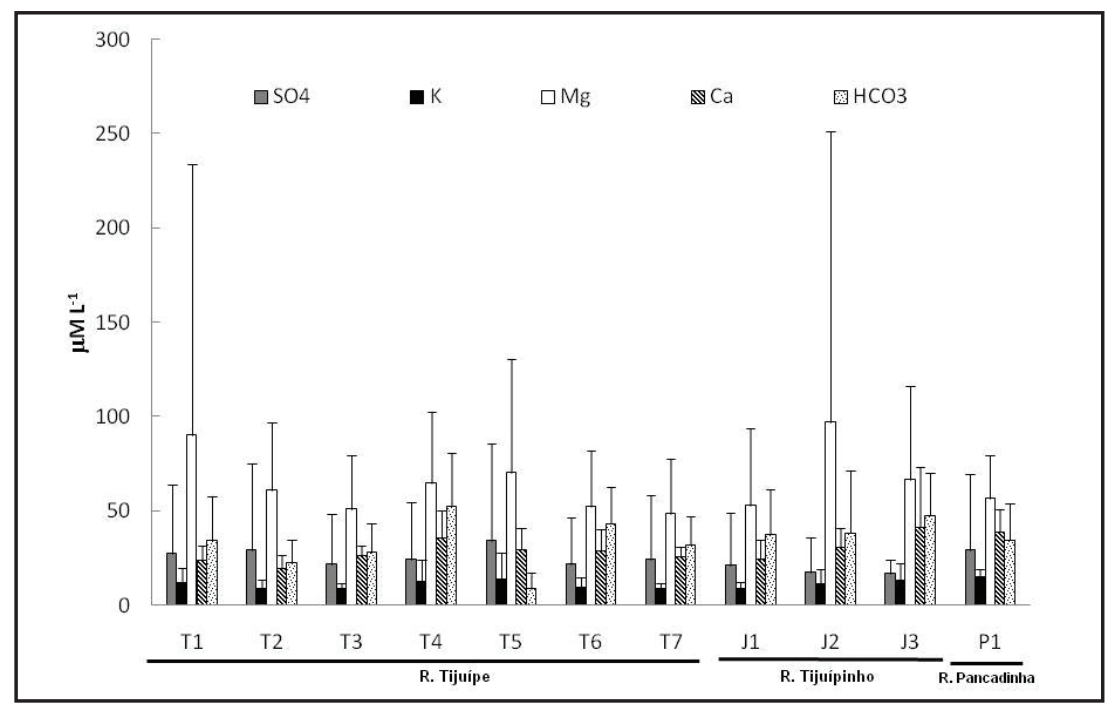

Figura 4 - Distribuição espacial dos íons maiores $\left(\mathrm{SO}_{4}^{2-}, \mathrm{K}^{+}, \mathrm{Mg}^{2+}, \mathrm{Ca}^{2+}\right.$ e $\left.\mathrm{HCO}_{3}^{-}\right)$ao longo dos pontos de coleta na bacia hidrográfica do rio Tijuipe (Uruçuca, Bahia)

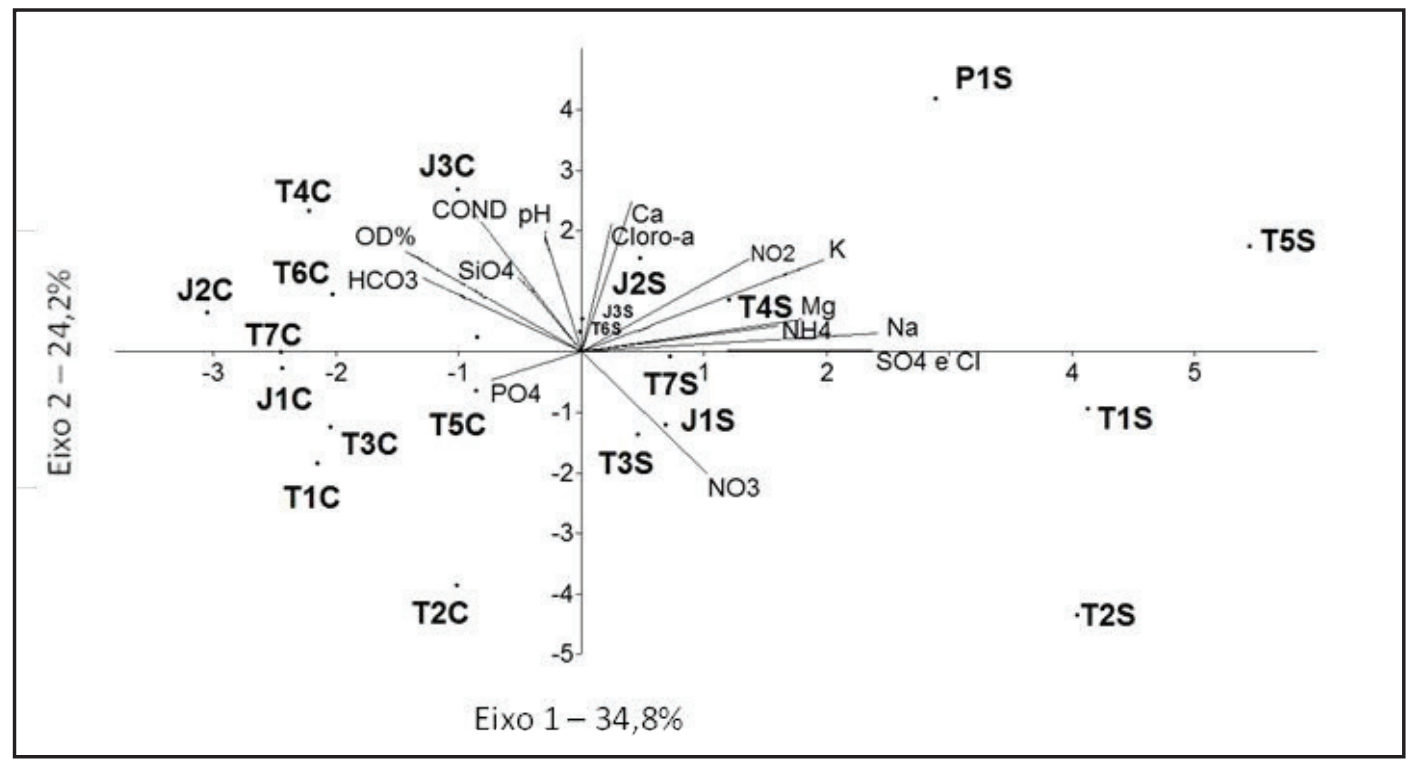

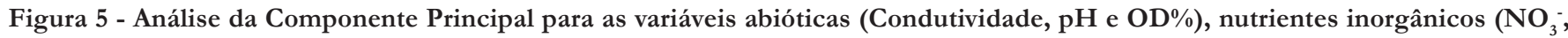

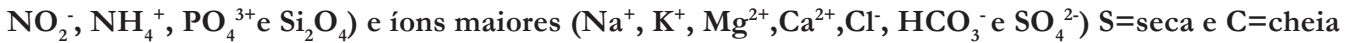




\section{DISCUSSÃO}

Diversos fatores são responsáveis por alterações que ocorrem ao longo de uma bacia de drenagem, entre eles estão a litologia da região, o tipo de vegetação circundante, o uso do solo e a proximidade com áreas urbanas. No caso da bacia do Rio Tijuipe, era esperado que o ponto P1 localizado no rio Pancadinha apresentasse valores distintos dos demais por estar localizado em uma área mais urbanizada. No entanto, a não ser pelas maiores concentrações de SST e condutividade, não foram encontradas diferenças estatisticamente significativas em relação aos demais pontos localizados em áreas preservadas, sugerindo assim que a influencia das modificações no uso do solo neste caso não altera a biogeoquímica desse rio.

A presença de mata ciliar no entorno da bacia hidrográfica é determinante para o comportamento dos nutrientes nos rios. As matas ciliares são efetivas na remoção de diversos íons e desempenham um papel crucial nas trocas de água e de elementos químicos entre os ecossistemas terrestres e aquáticos (BURT; PINAY 2005; GREGORY et al., 1991). De acordo com Vidon e Hill (2004), o fluxo de nutrientes em matas ciliares apresenta um forte controle dos fluxos laterais, expressos pelo escoamento superficial e subsuperficial. No presente estudo, o silicato e o nitrato parecem ser regulado pela presença da mata ciliar em alguns pontos de coleta.

A presença da mata ciliar nos pontos a montante de cada rio influencia na menor concentração desses nutrientes nestes locais devido a vegetação exercer uma função de filtro, reduzindo assim a lixiviação deste íon aos corpos d'água (HILL; DEVITO; VIDON, 2014; HUMBORG et al., 2006; MEUNIER et al., 2015). Resultado semelhante foi encontrado por Marques, Oliveira e Machado (2003) no Rio Piraquara, que revelou um considerável incremento nas concentrações de silicato após o rio atravessar áreas onde a vegetação ciliar foi retirada.

Este comportamento não foi observado no Rio Tijuipe onde não houve necessariamente uma redução nos valores desse nutriente da montante e jusante. De acordo com Hill, Devito e Vidon (2014) a eficiência na remoção de nutrientes (principalmente nitrato) pelas matas ciliares estão associadas ao tempo de residência que permite a interação entre a água subterrânea e os sítios de retenção bióticos nos primeiros metros da mata ciliar. A denitrificação pode também ser responsável pelos menores valores encontrados a montante do Rio Tijuipinho. Este processo ocorre com frequência em áreas com lençol freático mais superficial e solos ricos em matéria orgânica associados á baixa perda de N na interface solo-água (HANSON; GROFFMAN; GOLD, 1994)

O rio Tijuipe apresentou os maiores valores de nitrato ao longo de toda a bacia, principalmente nos pontos T1 e T2. Estes pontos estão localizados em riachos de primeira ordem localizado em uma floresta ombrófila cujo tempo de residência da água provavelmente é suficiente para permitir sua interação com a água subterrânea resultando em um incremento nas concentrações de nitrato proveniente da decomposição de matéria orgânica no solo e na água (HILL; DEVITO; VIDON, 2014). É provável que nestes pontos de coleta a entrada de $\mathrm{N}$ via água subterrânea exceda a necessidade de $\mathrm{N}$ pelo solo e plantas e os processos de perdas de N, tais como, denitrificação, sejam reduzidos.

Resultados similares foram encontrados por Andrade et al. (2011) e Silva et al. (2012) em riachos de áreas preservadas no Sudeste brasileiro, onde o nitrato foi a forma predominante no corpo d'água. Tal comportamento pode ser observado através da ACP onde pode ser verificado que os pontos a montante do rio Tijuipe (T1 e T2), para ambas as estações, mostraram um padrão diferenciado dos trechos médio e final dos rios, sendo encontrados nos quadrantes inferiores (quadrantes III e IV). Este gradiente espacial está associado ao eixo 1, tendo como principal forçante a variável $\mathrm{NO}_{3}$.

A predominância de nitrato, em relação às demais formas de nitrogênio inorgânico dissolvido, ocorre em virtude deste íon possuir maior mobilidade no solo aumentando sua capacidade de ser lixiviado, com consequente aumento nos corpos d'água, além de ser também a forma mais estável de nitrogênio. Temporalmente o nitrato apresentou a maior variação $(0,49-9,30$ $\mu \mathrm{M})$ nos meses secos em todos os pontos de coleta, exceção aos pontos T1 e T2 que apresentaram um aumento no aporte nos períodos mais chuvosos respondendo rapidamente a eventos de chuva, sendo facilmente lixiviado em função da intensidade do evento (CHEN; HONG, 2011). Mesmo padrão foi observado por Evans et al. (2014) que atribuiu este comportamento ao aumento das conexões hidrológicas durante o período chuvoso entre as fontes de nitrato que ficaram acumulados nos períodos mais secos nos ecossistemas terrestres.

Outro fator que também pode ter influenciado na concentração de nitrato foi a supersaturação de oxigênio, uma vez que o processo de nitrificação é aeróbio. As altas concentrações de oxigênio, como observado nas águas da bacia, favorecem a presença de formas oxidadas de todos os íons no sistema.

O fósforo foi encontrado em baixas concentrações na maioria dos pontos de coleta. Este elemento representa um dos principais componentes de diversos minerais, tais como, fosfato de cálcio, fosfato de ferro e fosfato de alumínio e se liga facilmente a argilas silicatadas, material húmico, ferro e óxidos de alumínio. Estas ligações químicas inibem a liberação de fósforo do solo e sedimento tornando baixa a disponibilidade deste na coluna d'água (LIKENS, 2010).

A diferença na composição das águas mundiais em relação ao conteúdo iônico refere-se basicamente às concentrações de $\mathrm{Ca}^{2+}, \mathrm{HCO}_{3}{ }^{-}$e $\mathrm{Na}^{+}$(VIEIRA; MOURA; FERREIRA, 2005). Cole (1983) e Wetzel (1991) demonstraram que a tendência mundial obedece à ordem $\mathrm{Ca}^{2+}>\mathrm{Mg}^{2+}>\mathrm{Na}^{+}>\mathrm{K}^{+}$, no entanto, esta sequência pode sofrer alterações em decorrência de características da bacia de drenagem, da composição dos sedimentos aquáticos e da climatologia local.

Os íons $\mathrm{Na}^{+}$e $\mathrm{Cl}^{-}$apresentaram as maiores concentrações entre os íons analisados, para todos os rios da bacia, sendo isto uma característica de rios próximo à costa (GIBBS, 1970).

A razão $\mathrm{Na}^{+} /\left(\mathrm{Na}^{+}+\mathrm{Ca}^{2+}\right)$ é utilizada para verificar qual é o mecanismo predominante na composição química da água. Quando a razão se aproxima de 1 a chuva é a principal fonte de sais para as águas dos rios, e quando a razão diminui o mecanismo regulador é o intemperismo das rochas pela água subterrânea (DREVER, 1997). A média da bacia hidrográfica 
do rio Tijuipe foi de 0,90 , deste modo o mecanismo regulador da composição química é a água da chuva. Segundo Lewis Jr. (1981) a precipitação é uma das principais fontes de nutrientes e íons para ecossistemas aquáticos e terrestres, sendo que a natureza de seus constituintes químicos depende da qualidade da fonte, da direção dos ventos, da proximidade do mar e das atividades do uso do solo.

Além disso, a razão $\mathrm{Cl} / \mathrm{Na}$ no presente trabalho apresentou um valor médio para a bacia de 1,09 $\pm 0,12$. Esta razão média, quando comparada com a razão da água do mar $(1,17)$ indica um excesso de $\mathrm{Na}^{+}$, que pode ser oriundo de fontes continentais (BERNER; BERNER, 1987), ou um déficit de $\mathrm{Cl}^{-}$no ambiente. Resultados semelhantes foram obtidos por Flues et al. (2002) e por Mello (2001) em trabalhos que avaliavam a quantidade de íons presentes na água da chuva no Estado do Paraná e no Rio de Janeiro, respectivamente, onde foi observado um menor valor desta razão devido a influência de fontes continentais. Vale ressaltar que o ponto T1 apresentou valor superior para esta razão $(1,19 \pm 0,76)$, possivelmente associado à composição da deposição atmosférica, que pode sofrer influência de fontes marinhas por vários quilômetros no continente, a depender do regime de ventos e do relevo da região.

Através da analise dos dados da ACP é possível perceber a existência de uma separação temporal entre os pontos, com o gradiente sazonal associado ao eixo 2 , e influenciado positivamente pelos íons maiores cloreto, sulfato e sódio e negativamente pelo bicarbonato e pelo OD \%. Essa correlação entre o período de seca e os íons maiores é devido aos elevados valores encontrados para todos os íons, com exceção do cálcio e do bicarbonato, durante os meses mais secos (outubro e novembro) (Figura 5). Este padrão já era esperado, uma vez que as variações nas concentrações de cátions e ânions estão diretamente relacionadas a flutuações nas vazões dos rios (CAMERON et al., 1995). Segundo Gibbs (1970), o principal mecanismo regulador da química das águas em rios é o intemperismo químico das rochas, que está relacionado com o maior tempo de residência das águas subterrâneas com o substrato rochoso no período de seca, aumentando, assim, aportes via intemperismo químico. Além disso, é no período chuvoso que ocorre a diluição das concentrações dos íons na água dos rios, devido ao maior aporte de água da chuva, que é menos rica em sais. Essa diluição dos íons nos períodos mais chuvosos tem sido observados em estudos em riachos em diversas áreas (HORBE et al., 2005; KAMISAKO et al., 2008, SILVA, 2000; YUSOP; DOUGLAS; NIK, 2006) sendo que esta variação temporal relacionada com a diluição do fluxo de base e/ou das fontes antropogênicas.

\section{CONCLUSÃO}

A vegetação de Mata Atlântica localizada na APA Itacaré Serra-Grande e Parque Estadual da Serra do Conduru exerce papel importante na preservação das características geoquímicas da Bacia do Rio Tijuipe. A mata ciliar em alguns pontos de coleta demonstrou eficiência na retenção de nutrientes tais como nitrato e silicato.
A precipitacao ao longo da bacia hidrográfica apresentou influência sobre a concentração dos íons maiores, com os menores valores observados para os meses mais chuvosos, devido ao processo de diluição.Esta relação foi observada através da ACP que revelou que os pontos da bacia hidrográfica encontram-se divididos entre as estações, com os meses de seca sendo influenciados pelos íons $\mathrm{Cl}^{-}, \mathrm{Na}^{+}$e $\mathrm{SO}_{4}^{3-}$ e os meses de cheia com influencia do bicarbonato e do OD $\%$.

O rio Pancadinha é o único ponto que possui residências em suas margens e apesar de ter apresentado valores de SST e condutividade superiores aos demais rios, não demonstrou diferenças significativas em relação aos íons analisados indicando que os impactos não alteraram a ciclagem biogeoquímica destes íons.

A bacia hidrográfica do rio Tijuipe possui uma influência direta dos aerossóis marinhos que podem ser confirmados através das maiores concentrações dos íons $\mathrm{Cl}^{-}$e $\mathrm{Na}^{+}$predominantes nos três rios estudados.

\section{AGRADECIMENTOS}

Os autores agradecem a FAPESB (Projeto PPP TO 0040/2011 e Bolsa de Mestrado) e CNPq (INCT - TMCOcean) pelo auxílio financeiro e à UESC pela contrapartida para as coletas de campo.

\section{REFERÊNCIAS}

ANDRADE, T. M. B.; CAMARGO, P. B.; SILVA, D. M. L.; PICCOLO, M. C.; VIEIRA, S. A.; ALVES, L. F.; JOLY, C. A.; MARINELLI, L. A. Dynamics of dissolved forms of carbon and inorganic nitrogen in small watersheds of the Coastal Atlantic Forest in Southeast Brazil. Water, Air, Soil Pollut., v. 214, n. 1-4, p. 393-408, Jan. 2011.

ASMAR, S. R.; ANDRADE, M. P. Geografia da Microrregião Cacaueira. Itabuna - BA: Comissão Executiva do Plano da Lavoura Cacaueira - CEPLAC, 1977.

BERNER, E. K.; BERNER, R. A. The Global Water Cycle: geochemistry and environment. In: BERNER, E. K.; BERNER, R. A. Rainwater and Atmospheric Chemistry. New Jersey: Prentice-Hall; Englewood Cliffs, 1987. cap. 3.

BURT, T. P.; PINAY, G. Linking hydrology and biogeochemistry in complex landscapes. Prog. Phys. Geogr., v. 29, n. 3, p. 297-316, Sept. 2005.

BRASIL, Decreto No2186 DE 07 DE JUNHO DE 1993. Dispõe sobre a criação da Área de Proteção Ambiental da Costa de Itacaré/Serra Grande.

CAMERON, E. M.; HALL, G. E. M.; VEIZER, J.; KROUSE, H. R. Isotopic and Elemental hydrogeochemistry of major river system: Fraser River, British Columbia, Canada. Chem. Geol., v. 
122, n. 1-4, p. 149-169, May 1995.

CARMOUZE, J. P. O Metabolismo dos Ecossistemas Aquáticos: - fundamentos teóricos, métodos de estudo e análises químicas. São Paulo: Edgard Blücher; FAPESP, 1994.

CHEN, N.; HONG, H. Nitrogen export by surface runoff from a small agricultural watershed in southeast China: seasonal pattern and primary mechanism. Biogeochem., v. 106, n. 3, p. 311321, Nov. 2011.

COLE, G. A. Textbook of limnology. 3. ed. London: C.V. Mosby, 1983.

DEEGAN, L. A.; NEILL, C.; HAUPERT, C. L.; BALLESTER, M. V. R.; KRUSCHE, A. V.; VICTORIA, R. L.; THOMAS, S. M.; MOOR, E. Amazon deforestation alters small stream structure, nitrogen biogeochemistry and connectivity to larger Rivers. Biogeochem., v. 105, n. 1-3, p. 53-74, Sept. 2011.

DEFRIES, R.; ESHLEMAN, N. K. Land-use change and hydrologic processes: a major focus for the future. Hydrol. Process., v. 18, n. 11, p. 2183-2186, Aug. 2004.

DREVER, J. I. The carbonate system and $\mathrm{pH}$ control. In: DREVER, J. I. The geochemistry of natural waters: surface and groundwater environments. 3. ed. Englewood Cliffs: Prentice Hall, 1997. p. 41-68.

EVANS, D. M.; SCHOENHOLTZ, S. H.; WIGINGTON JR, P. J.; GRIFFITH, S. M.; FLOYD, W. C. Spatial and temporal patterns of dissolved nitrogen and phosphorus in surface waters of a multi-land use basin. Environ. Monit. Assess., v. 186, n. 2, p. 873-887, Feb. 2014.

FLUES, M.; HAMA, P.; LEMES, M. J. L.; DANTAS, E. S. K.; FORNARO, A. Evaluation of the rainwater acidity of a rural region due to a coal-fired power plant in Brazil. Atmos. Environ., v. 36, n. 14, p. 2397-2404, May 2002.

GALINDO-LEAL, C.; CÂMARA, I. G. (Ed.). Mata Atlântica Biodiversidade, Ameaças e Perspectivas. Belo Horizonte: Fundação SOS Mata Atlântica e Conservação Internacional, 2005. p. 119-132.

GIBBS, J. R. Mechanisms controlling world water chemistry. Science, v. 170, n. 3962, p. 1088-1090, Dec. 1970.

GRASSHOFF, K.; EHRARDT, M.; KREMLING, K. Methods of seawater analysis. Wheinhein: Verlag Chermie, 1983.

GREGORY, S. V.; SWANSON, F. J.; MCKEE, W. A.; CUMMINS, $\mathrm{K}$. W. An ecosystem perspective of riparian zones. Bioscience, v. 41, n. 8, p. 540-551, Sept. 1991.

HANSON, G. C.; GROFFMAN, P. M.; GOLD, A. J. Symptoms of nitrogen saturation in a riparian wetland. Ecol. Appl., v. 4, p. $750-756,1994$.
HILL, A. R.; DEVITO, K. J.; VIDON, P. G. Long-term nitrate removal in a stream riparian zone. Biogeochemistry, v. 121, n. 2, p.425-439, Nov. 2014.

HORBE, A. M. C.; GOMES, I. L. F.; MIRANDA, S. F.; SILVA, M. S. R. Contribuição à hidroquímica de drenagens no Município de Manaus - AM. Acta Amazonica, v. 35, n. 2, p. 119-124, abr./ jun. 2005.

HOWARTH, R.; ANDERSON, D.; CLOERN, J.; ELFRING, C.; HOPKINSON, C.; LAPOINTE, B.; MALONE, T.; MARCUS, N.; MCGLATHERY, K.; SHARPLEY, A.; WALKER, D. Nutrient pollution of coastal rivers, bays, and seas. Issues Ecol., v. 7, p. 1-15, Fall 2000.

HUMBORG, C.; PASTUSZAK, M.; AIGARS, J.; SIEDMUND, H.; MORTH, C. M.; ITTEKKOT, V. Decreased silica land-sea fluxes through damming in the Baltic Sea catchment: significance of particle trapping and hydrological alterations. Biogeochemistry, v. 77, n. 2, p. 265-281, Feb. 2006.

IESB - INSTITUTO DE ESTUDOS SOCIO AMBIENTAIS DO SUL DA BAHIA. Plano de Manejo do Parque Estadual da Serra do Conduru (PESC). [S.1.]: IESB, 2005.

KAMISAKO, M.; SASE, H.; MATSUI, T.; SUZUKI, H.; TAKAHASHI, A.; OIDA, T.; NAKATA, M.; TOTSUKA, T.; UEDA, T. Seasonal and Annual Fluxes of Inorganic Constituents in a Small Catchment of a Japanese Cedar Forest near the Sea of Japan. Water Air Soil Pollut., v. 195, n. 1-4, p. 51-61, Nov. 2008.

LEWIS, E.; WALLACE, D. W. R. Program Developed for CO2 System Calculations. ORNL/CDIAC- 105. Oak Ridge, Tennessee: Carbon Dioxide Information Analysis Center, Oak Ridge National Laboratory, U.S. Department of Energy, 1998.

LEWIS, Jr., W. M. Precipitation Chemistry and Nutrient Loading by Precipitation in a Tropical Watershed. Water Resour. Res., v. 17, n. 1, p. 169-181, Feb. 1981.

LIKENS, G. E. Biogeochemistry of inland waters. New York: Elsevier, 2010. 745 p.

MARQUES, P. H. C.; OLIVEIRA, H. T.; MACHADO, E. C. Limnological study of Piraquara River (upper Iguaçu basin): spatiotemporal variation of physical and chemical variables and watershed zoning. Bra\%. Arch. Biol. Technol. Int. J., v. 46, n. 3, p. 383-394, June 2003.

MELLO, W. Z. Precipitation Chemistry in the Coast of the Metropolitan Region of Rio de Janeiro, Brazil. Environ. Pollut., v. 114, n. 2, p. 235-242, 2001.

MEUNIER, J. D.; RIOTTE, J.; BRAUN, J. J.; SEKHAR, M.; CHALIÉ, F.; BARBONI, D.; SACCONE, L. Controls of DSi in streams and reservoirs along the Kaveri River, South India. Sci. Total Environ., v. 502, p. 103-113, Jan. 2015. 
MYERS, N.; MITTERMEIER, R. A.; MITTERMEIER, C. G.; FONSECA, G. A. B.; KENT, J. Biodiversity hotspots for conservation priorities. Nature, v. 403, n. 6772, p. 853-858, Feb. 2000 .

PARRON, L. M.; BUSTAMANTE, M. M. C.; MARKEWITZ, D. Fluxes of nitrogen and phosphorus in a gallery forest in the Cerrado of central Brazil. Biogeochemistry, v. 105, n. 1-3, p. 89-104, Sept. 2011.

PFAFF, J. D.; HAUTMAN, D. P.; MUNCH, D. J. USEPA method 300.1. Determination of inorganic anions in drinking water by ion chromatography. Cincinnati, Ohio: National Exposure Research Laboratory, Office of Research and Development, U.S. Environmrntal Protection Agency, 1997.

PNUMA. Portal do Núcleo da Mata Atlântica. [S.l.: s.n.], 2012. Disponível em: <http://mpnuma.ba.gov.br/index. php?option $=$ com_Content $\&$ view $=$ article $\& i d=59 \&$ Itemid $=75>$. Acesso em: 13 abr. 2012.

PROCLIMA, Programa de Monitoramento Climático em Tempo Real da Região Nordeste. Disponível em: http://www6.cptec. inpe.br/proclima2/balanco_hidrico/balanco hidrico.shtml. Acesso em Janeiro de 2012.

SÁ, D. F.; ALMEIDA, H. A.; LEÃO, A. C. Fatores Edafoclimáticos Seletivos ao Zoneamento de Cacauicultura no Sudeste da Bahia. Theobroma, v. 12, n. 3, p. 169-187, jul./set. 1982.

SILVA, C. F. Hidrogeoquímica fluvial da porção inferior do rio Paraíba do Sul, RJ. 2000. 103 f. Dissertação (Mestrado) - Centro de Biociências e Biotecnologia, Universidade Estadual do Norte Fluminense, Campos dos Goytacazes, Rio de Janeiro, 2000.

SILVA, D. M. L.; CAMARGO, P. B.; MCDOWELL, W. H.; VIEIRA, I.; SALOMÃO, M. S. M. B.; MARTINELLI, L. A. Influence of land use changes on water chemistry in streams in the State of São Paulo, southeast Brazil. Anais Acad. Bras. Ciênc., v. 84, n. 4, p. 919-930, dez. 2012.

SILVA, L. P. Dinâmica de nitrogênio em microbacias em remanescentes de mata atlântica (Itacaré-Uruçuca/Ba). 2012. 67 f. Dissertação (Pós-graduação em Sistemas Aquáticos Tropicais) - Universidade Estadual de Santa Cruz, Ilhéus, Bahia, 2012.

SOS MATA ATLÂNTICA. Atlas da Mata Atlântica. [S.l.: s.n.], 2013. Disponível: <http://www.sosma.org.br/14622/ divulgados-novos-dados-sobre-a-situacao-da-mata-atlantica/>. Acesso em: 5 jun. 2013.

SYVITSKI, J. P. M.; VÖRÖSMARTY, C. J.; KETTNER, A. J.; GREEN, P. Impact of Humans on the Flux of Terrestrial Sediment to the Global Coastal Ocean. Science, v. 308, n. 5720, p. 376-380, Apr. 2005.

VIDON, P. G. F.; HILL, A. R. Landscape controls on the hydrology of stream riparian zones. J Hydrol., v. 292, n. 1-4, p. 210-228, June 2004.

VIEIRA, M. S.; MOURA, M. A. M.; FERREIRA, J. R. Estudo das variações sazonais e espaciais dos íons dominantes na água e no sedimento da represa de Ibitinga (São Paulo, Brasil - 21 ${ }^{\circ} 45^{\prime} \mathrm{S}$ e 4850’W). Arq. Inst. Biol., v. 72, n. 4, p. 523-534, out./dez., 2005.

WETZEL, R. A. Limnological analyses. 2. ed. New York: Springer-Verlag, 1991.

YUSOP, Z. A.; DOUGLAS, I. B.; NIK, A. R. Export of dissolved and undissolved nutrients from forested catchments in Peninsular Malaysia. Forest Ecol. Management v. 224, n. 1-2, p. 26-44, Mar. 2006. 